Mathematical Research Letters 5, 637-655 (1998)

\title{
ON THE TATE-SHAFAREVICH GROUP OF CERTAIN ELLIPTIC CURVES
}

\section{Farshid Hajir and Fernando Rodriguez Villegas}

Let $K \subset \mathbb{C}$ be an imaginary quadratic field with prime discriminant $-p<-3$, ring of integers $\mathcal{O}_{K}=\mathcal{O}$ and class group $\mathrm{Cl}_{K}$ of (odd) order $h_{K}=h$. The $j$ invariant $j(\mathcal{O})$ generates a field $F / \mathbb{Q}$ of degree $h$ such that $H=F K$ is the Hilbert class field of $K$. Suppose $A / F$ is a $\mathbb{Q}$-curve with $j$-invariant $j(\mathcal{O})$; thus, $A$ is an elliptic curve which, over $H$, is isogenous to each of its Galois conjugates, and has complex multiplication by $\mathcal{O}$. We let $B=\operatorname{Res}_{F / \mathbb{Q}} A$ be the $h$-dimensional abelian variety over $\mathbb{Q}$ obtained from $A$ by restriction of scalars. Any two such $A$ (and any two such $B$ ) are quadratic twists of one another: letting $A(p)$ denote the canonical curve of discriminant ideal $\left(-p^{3}\right)$, with restriction $B(p)$, we have $A=A(p)^{D}$ (and $\left.B=B(p)^{D}\right)$ for some quadratic discriminant $D$. We refer the reader to Gross [Gr1] for general facts about $\mathrm{CM} \mathbb{Q}$-curves. Much progress has been made recently on proving the conjecture of Birch and Swinnerton-Dyer for these curves. For instance, if $L(1, A / F) \neq 0$, and $h=1$, Rubin [Ru2] has proved that the Birch-Swinnerton-Dyer conjecture for $A / F$ holds up to a power of 2. Note that the ring $\mathcal{R}^{+}$of $\mathbb{Q}$-endomorphisms of $B$ (or $B(p)$ ) is an order in $T^{+}=\mathcal{R}^{+} \otimes \mathbb{Q}$, a totally real field of degree $h$ over $\mathbb{Q}$. The Tate-Shafarevich group $\coprod_{B / \mathbb{Q}}$ is a finite module over $\mathcal{R}^{+}$; our main goal in this paper is to gain insight into the structure of this module via $L$-series in some special cases.

Namely, suppose $p \equiv 3 \bmod 8$ and $A=A(p)^{-3}$. Also, assume that $\mathcal{R}^{+}$is integrally closed. Let $\psi$ be a Hecke character of $K$ such that $\psi \circ \mathbb{N}_{H / K}$ is the Hecke character attached to $A / H$. This choice of $\psi$ gives rise to an embedding of $T^{+}$in $\mathbb{R}$ (see section 2). We define an algebraic integer $s \neq 0$ in $F T^{+}$as a sum of certain modified elliptic units first introduced by Gross [Gr3] and show that there is a (unique) integral ideal $\mathfrak{f}$ of $\mathcal{R}^{+}$whose lift to $\mathrm{FT}^{+}$is generated by $s$. Our starting point is a formula (Theorem 2) expressing $L(1, \psi)$ as a period times $s^{2}$, showing, in particular, that this central critical value does not vanish. Writing $L(s, A / F)$ as a product of Hecke L-series, calculating the local factors in the Birch-Swinnerton-Dyer conjecture, and applying our formula together with results of Coates, Wiles, Arthaud, and Rubin [CW], [Ar], [Ru1], we obtain

Main Theorem(Theorem 5) With the above assumptions and notation, $A(F)=$ $B(\mathbb{Q})$ is finite. If the Birch-Swinnerton-Dyer conjecture holds for $A / F$ (or for

Received September 19, 1997, revision received May 4, 1998.

The first author was supported in part by a Mathematical Sciences Postdoctoral Research Fellowship from the NSF. The second author was supported in part by a grant from the NSF and by a fellowship from the John Simon Guggenheim Memorial Foundation. 
$B / \mathbb{Q})$, then the order of its Tate-Shafarevich group is $\mathbb{N}_{T^{+} / \mathbb{Q}}(\mathfrak{f})^{2}$, where $\mathfrak{f}$ is the integral $\mathcal{R}^{+}$-ideal defined in section 2.

Following Buhler-Gross [BG], we conjecture that the order ideal $\mathfrak{s}_{B}$ of the $\mathcal{R}^{+}$module $\coprod_{B / \mathbb{Q}}$ is in fact $\mathfrak{f}^{2}$; note that, by our theorem, this is compatible with the Birch-Swinnerton-Dyer conjecture, and is a refinement of it. Our results shore up the Buhler-Gross conjecture in several ways: namely, our predicted order ideal $\mathfrak{f}^{2}$ is known to be the square of an integral ideal of $\mathcal{R}^{+}$. Previously, Buhler and Gross verified these properties numerically in hundreds of cases.

Our formula gives an effective means of computing the (predicted) order ideal of the Tate-Shafarevich group for these curves, and is similar to the formula one of us $[\mathrm{RV}]$ found for the cardinality of $\coprod_{A(p) / F}$ for $p \equiv 7 \bmod 8$; see also $[\mathrm{H}]$. We implemented this procedure on a computer (some tables of our data appear at the end of the paper). This allowed us to make a conjectural numerical study of the $\mathcal{R}^{+}$-module $\coprod_{B / \mathbb{Q}}$. We were particularly interested in the question of whether this module can be "non-trivial," in the sense that its order ideal $\mathfrak{s}_{B}$ is not trivial in the class group of $\mathcal{R}^{+}$. We succeeded in finding three examples where the (predicted) order ideal $\mathfrak{f}^{2}$ is not principal, not an entirely trivial task. Using some results from $[\mathrm{H}]$, this answers, at the same time, a question of Gross concerning the triviality of a certain 1-cocycle induced by the units used to define $\mathfrak{f}$. We should remark that there are order ideals attached to the torsion of $B / \mathbb{Q}$ and to its local factors at infinity and at the places of bad reduction (see Gross [Gr2]), and that a suitable product of all these ideals with the order ideal of the Tate-Shafarevich group is expected to be principal (generated by the algebraic part of the critical L-value), but that these ideals need not be principal in $\mathcal{R}^{+}$ individually, as demonstrated here.

We should explain that the restriction to the rather special case of twisting by -3 is made in order to keep the technicalities in the calculation of $L(1, \psi)$ (in section 5) to a minimum. The general factorization formula we prove in that section (Proposition 8 ) should yield a similar formula for $L(1, \psi)$ for more general twists, but in the case of twisting by -3 , the various Jacobi theta functions with characteristics reduce down to the relatively simple eta function; moreover, in the calculation of the local factors in the Birch-Swinnerton-Dyer conjecture, various dyadic primes just cancel in the case of twist by -3 , giving the clean formula for the predicted order ideal presented here. Also, see the recent paper by Rodriguez Villegas and Yang [RVY].

The organization of the sections is as follows. We set up the notation and make various definitions in section 1 . In section 2 , we define the algebraic quantities $s$ and $\mathfrak{f}$; the action of Galois on them is determined easily using the results of [HRV]. In section 3 , we introduce the elliptic curves $A$ of interest, and obtain a formula for $L(1, A / F)$, assuming the formula for $L(1, \psi)$ (whose proof, being somewhat technical, we defer to section 5). A calculation of the various factors in the Birch-Swinnerton-Dyer conjecture then yields the main theorem. In section 4, we recall the question of Gross, first posed in [Gr3] and further investigated in $[\mathrm{H}]$, and present calculations carried out using the package GP- 
PARI, illustrating three examples where the class of the (predicted) order ideal of the Tate-Shafarevich group is non-trivial, answering Questions 2.9, 2.10 and 2.13 of $[\mathrm{H}]$. As in [RV], the two main ingredients for expressing $L(1, \psi)$ as a period times $s^{2}$ are, first a formula, following Hecke, expressing $L(1, \psi)$ in terms of binary theta series (section 5.1), and a "factorization formula" expressing values of these theta series at CM points as a product of values of two half-integralweight theta series at related CM points (section 5.2). We conclude with a table of predicted orders of the Tate-Shafarevich group of $A(p)^{-3}$, as well as a table of the ideals $\mathfrak{f}$ for small $p$.

\section{Preliminaries}

Suppose $K \subset \mathbb{C}$ is an imaginary quadratic field with discriminant $-d$ relatively prime to 6 and class number $h$. With the exception of sections 1 and 5 , we will in fact assume $d=p$ is a prime $\equiv 3 \bmod 8$. (At the top of each section, we indicate our assumptions regarding $d$ ). We write $\mathcal{O}=\mathcal{O}_{K}$ for the ring of integers of $K$. Let $\epsilon$ be the Dirichlet character associated to $K$, extended to $K$ via the natural isomorphism $\mathcal{O} /(\sqrt{-d}) \cong \mathbb{Z} / d \mathbb{Z}$; concretely,

$$
\epsilon(\alpha)=\left(\frac{2(\alpha+\bar{\alpha})}{d}\right) \quad\left(\alpha \in \mathcal{O}_{K}\right)
$$

Here and throughout the paper, we use the notation $(\div)$ for the Kronecker symbol. We write $\left(\frac{2}{d}\right)=(-1)^{\delta}$ with $\delta=0,1$. Also, define

$$
\kappa=\frac{3-\epsilon(3)}{2}= \begin{cases}1 & d \equiv 2 \bmod 3 \\ 2 & d \equiv 1 \bmod 3 .\end{cases}
$$

Consider the set $\Phi$ of Hecke characters $\phi$ of $K$ such that

$$
\phi((\alpha))=\epsilon(\alpha) \alpha, \quad \alpha \in \mathcal{O}_{K}, \quad(\alpha, d)=1 .
$$

There are exactly $h$ such characters, they all have conductor $(\sqrt{-d})$, the ratio of any two being a character of $\mathrm{Cl}_{K}$. Let $\chi$ be the quadratic Dirichlet character associated to $\mathbb{Q}(\sqrt{-3})$,i.e. $\chi(a)=\left(\frac{-3}{a}\right)=\left(\frac{a}{3}\right)$ for integers $a$ prime to 3 and $\chi(a)=0$ otherwise. Consider the set $\Psi$ of twists $\psi=\phi \cdot \chi \circ \mathbb{N}_{K / \mathbb{Q}}$ with $\phi \in \Phi$. If $\mathfrak{a}$ is not relatively prime to $3 d, \psi(\mathfrak{a})=0$, and, for all $\mathfrak{a}, \psi(\overline{\mathfrak{a}})=\overline{\psi(\mathfrak{a})}$. Also,

$$
\bar{\psi}(\mathfrak{a}) \psi(\mathfrak{a})=\mathbb{N a} .
$$

For the remainder of the paper, fix a base point $\phi_{0} \in \Phi$ and its $\chi$-twist $\psi_{0} \in \Psi$; their unramified twists $\phi_{0} \varphi, \psi_{0} \varphi-$ with $\varphi \in \widehat{\mathrm{Cl}_{K}}-\operatorname{span} \Phi, \Psi$, respectively.

We will need two kinds of CM-points attached to an ideal $\mathfrak{a}$ of $\mathcal{O}$ : one, denoted $\tau_{\mathfrak{a}}$, at which we will evaluate integral-weight modular forms (of level $d$ ), and another, denoted $z_{\mathfrak{a}}$, for half-integral-weight modular forms (of level a power of 2 ). For a primitive $\mathcal{O}$-ideal $\mathfrak{a}$ of norm $a$ prime to $6 d$, we may always choose a basis 
$\mathfrak{a}=a \mathbb{Z}+\frac{b+\sqrt{-d}}{2} \mathbb{Z}$ with $b \in \mathbb{Z}$ determined modulo $2 a$ satisfying $b^{2} \equiv-d \bmod 4 a$. In particular, we may choose $b \in 3 d \mathbb{Z}$, and put

$$
\tau_{\mathfrak{a}}=\frac{b+\sqrt{-d}}{2 a d} \in \mathcal{H} \quad\left(\mathfrak{a}=\left[a, \frac{b+\sqrt{-d}}{2}\right], \quad b \equiv 0 \bmod 3 d\right),
$$

which is well-defined modulo $3 \mathbb{Z}$ and whose image in $\mathcal{H} / \Gamma_{0}(d)$ depends only the class $[\mathfrak{a}]$. Similarly, we may always choose $b \equiv 1 \bmod 16$ and then set

$$
z_{\mathfrak{a}}=\frac{b+\sqrt{-d}}{2 a} \in \mathcal{H} \quad\left(\mathfrak{a}=\left[a, \frac{b+\sqrt{-d}}{2}\right], \quad b \equiv 1 \bmod 16\right),
$$

well-defined modulo $8 \mathbb{Z}$.

\section{The units $u_{C}$ and the ideal $f$}

In this section, we assume that $d=p>3$ is a prime satisfying $p \equiv 3 \bmod 8$, so $\delta=1$ and $h$ is odd. Let $j=j(\mathcal{O}) \in \mathbb{R}$ where $j(L)$ is the classical modular invariant of a lattice $L \subset \mathbb{C}$. Let $F=H^{+}=\mathbb{Q}(j) \subset \mathbb{R}$; this is a number field of degree $h$ whose remaining embeddings are complex. Recall that $H=H^{+} K$ is the Hilbert class field of $K$.

Let $T=T_{\phi_{0}}$ be the subfield of $\mathbb{C}$ generated by the values of $\phi_{0}$, and put $T^{+}=$ $T \cap \mathbb{R}$. Then $T^{+}$is totally real of degree $h$ over $\mathbb{Q}$ and $T / T^{+}$is a CM extension. Let $M^{+}=T^{+} H^{+}, M=M^{+} K$. Then $T \cap H=K$, and we may identify $\widehat{\mathrm{Cl}_{K}}$ with the embeddings of $M / H$ in $\mathbb{C}$, the trivial character corresponding to our fixed embedding $T_{\phi_{0}}$. For instance, for $x \in H, \varphi \in \widehat{\mathrm{Cl}_{K}}$, and $\mathfrak{a} \subseteq \mathcal{O}$, we have $\left(\phi_{0}(\mathfrak{a}) x\right)^{\varphi}=\left(\varphi \phi_{0}\right)(\mathfrak{a}) x$. Also, we may identify $\mathrm{Cl}_{K}$ with $\operatorname{Gal}(M / T)$ via the Artin map $C \mapsto \sigma_{C}$. For more detailed explanations of these facts, we refer the reader to Gross [Gr1].

Recall that the Dedekind eta function defined for $z \in \mathcal{H}$ by

$$
\eta(z)=e^{\pi i z / 12} \prod_{n \geq 1}\left(1-e^{2 \pi i z n}\right)
$$

has the series expansion

$$
\eta(z)=\sum_{m \geq 1}\left(\frac{12}{m}\right) e^{\pi i m^{2} z / 12}
$$

which converges quickly, when $\Im(z)$ is bounded below by a positive constant.

Definition 1. For each ideal class $C$, choose a primitive ideal a prime to $6 d$ such that $\left[\mathfrak{a}^{2}\right]=C^{-1}$ and put

$$
u_{C}=\left(\frac{-4}{\overline{N a}}\right) \overline{\phi_{0}}(\mathfrak{a})^{-1} \eta\left(z_{\mathfrak{a}^{2}}\right) / \eta\left(z_{\mathcal{O}}\right)
$$


Remark. That $u_{C}$ is well-defined may be checked directly; it also follows easily from Corollary 10 which we will establish in section 5 . For $d \equiv 7 \bmod 8$, the units $u_{C}$ were introduced already in [RV]; the definition in the two cases differs only by the factor $\left(\frac{-4}{\mathrm{Na}}\right)^{\delta}$. In the proof of $[\mathrm{H}$, Theorem 3.1], the presence of this symbol should have been mentioned.

To see the algebraic properties of $u_{C}$, we note that, in the notation of [HRV],

$$
\frac{\eta\left(z_{\mathfrak{a}^{2}}\right)}{\eta\left(z_{\mathcal{O}}\right)}=\frac{\eta\left(\overline{\mathfrak{a}}^{2}\right)}{\eta(\mathcal{O})}
$$

hence by [HRV, Prop. 10] $\eta\left(z_{\mathfrak{a}^{2}}\right) / \eta\left(z_{\mathcal{O}}\right) \in \mathcal{O}_{H}$ and generates $\overline{\mathfrak{a}} \mathcal{O}_{H}$. Since $\overline{\phi_{0}}(\mathfrak{a}) \in$ $\mathcal{O}_{T}$ and generates $\overline{\mathfrak{a}} \mathcal{O}_{T}, u_{C}$ is a unit in $M$. Moreover, by [HRV, Prop. 10],

$$
\bar{u}_{C}=u_{C^{-1}}, \quad u_{C}^{\sigma_{C^{\prime}}}=u_{C C^{\prime}} / u_{C^{\prime}} \quad\left(C, C^{\prime} \in \mathrm{Cl}_{K}\right) .
$$

We see that these units give rise to a 1-cocycle with values in $E_{M}=\mathcal{O}_{M}^{\times}$. In order to maintain consistency with the notation in $[\mathrm{H}]$, let us write for $\sigma=\sigma_{C} \in$ $\operatorname{Gal}(M / T), w_{\sigma}=u_{C}$. The assignment

$$
\sigma_{-} \mapsto 1, \sigma \mapsto w_{\sigma},
$$

(where $\sigma_{-}$is a generator of $\operatorname{Gal}\left(M / M^{+}\right)$and $\sigma \in \operatorname{Gal}(M / T)$ ) defines a 1-cocycle $w$ on $\operatorname{Gal}\left(M / T^{+}\right)$with values in $E_{M}$.

We let $s=\sum_{C \in \mathrm{Cl}_{K}} u_{C} \in \mathcal{O}_{M}$. It is easily verified that $\operatorname{Tr}_{M / H}(s)=h$, and that $\overline{s^{\varphi}}=s^{\varphi}$ for each $\varphi \in \widehat{\mathrm{Cl}_{K}}$; hence, $s^{\varphi}$ is a non-zero real number. Furthermore,

$$
s^{\sigma_{C}}=s u_{C}^{-1}, \quad\left(C \in \mathrm{Cl}_{K}\right) .
$$

In particular, the integral ideal $s \mathcal{O}_{M}$ is fixed by $\operatorname{Gal}(M / T)$. Since $M / T$ is unramified, and since $\bar{s}=s$, there is a unique ideal $\mathfrak{f}$ of $\mathcal{O}_{T^{+}}$such that $\mathfrak{f} \mathcal{O}_{M}=$ $s \mathcal{O}_{M}$. Note that $\mathfrak{f}^{h}$ is principal, generated by $s \prod_{C} u_{C} \in \mathcal{O}_{T^{+}}$; we will be interested in whether $\mathfrak{f}$ itself is principal in $\mathcal{O}_{T^{+}}$. We note that in $[\mathrm{H}], s$ and $\mathfrak{f}$ were called $s_{w}$ and $\mathfrak{f}_{w}$, respectively.

Section 5 will be devoted to the proof of

Theorem 2. For any unramified character $\varphi \in \widehat{\mathrm{Cl}_{K}}$,

$$
L\left(1, \psi_{0} \varphi\right)=\frac{4 \pi \kappa\left|\eta\left(z_{\mathcal{O}}\right)\right|^{2}}{3^{1 / 2} p^{1 / 4}}\left(s^{\varphi}\right)^{2} .
$$

\section{The $\mathbb{Q}$-curve $A(p)^{-3}$}

In this section, we continue to assume that $d=p>3$ is a prime satisfying $p \equiv 3 \bmod 8$. There is an elliptic curve $A(p)$ of invariant $j=j(\mathcal{O})$ with global minimal equation of discriminant $\left(-p^{3}\right)$ over $H^{+}$

$$
y^{2}=x^{3}+\frac{m p}{2^{4} 3} x-\frac{n p^{2}}{2^{5} 3^{3}},
$$


where $m, n$ are the unique real numbers defined by

$$
m^{3}=j, \quad-n^{2} p=j-1728, \quad n<0 .
$$

Using the classical theory of complex multiplication, one shows that $m, n$ are actually elements of $H^{+}$; see, for example, [HRV, Theorem 17]. Over $H, A(p)$ acquires complex multiplication by $\mathcal{O}_{K}$, and is isogenous to all of its Galois conjugates.

Consider the elliptic curve $A=A(p)^{\chi}=A(p)^{-3}$, the twist of $A(p)$ by $\mathbb{Q}(\sqrt{-3})$. The CM elliptic curve $A$ has associated Hecke character $\psi_{0} \circ \mathbb{N}_{H / K}$. By Shimura [Sh], we have the factorization

$$
L\left(s, A / H^{+}\right)=\prod_{\varphi \in \widehat{\mathrm{Cl}}_{K}} L\left(s, \psi_{0} \varphi\right) .
$$

Recall that $B=\operatorname{Res}_{H^{+} / \mathbb{Q}} A$ is the abelian variety over $\mathbb{Q}$ obtained from $A$ via restriction of scalars. It is an $h$-dimensional quotient of the Jacobian $J_{0}\left(9 p^{2}\right)$ of the modular curve $X_{0}\left(9 p^{2}\right)$. The $L$-series of $A / H^{+}$and $B / \mathbb{Q}$ coincide; the Birch-Swinnerton-Dyer conjecture holds for one if and only if it holds for the other, and $\coprod_{A / H^{+}}$and $\coprod_{B / \mathbb{Q}}$ are isomorphic, see Milne [Mi, Theorem 1]. The $\mathbb{Q}$-endomorphism ring $\mathcal{R}^{+}=\operatorname{End}_{\mathbb{Q}} B$ is an order in $T^{+}=\mathcal{R}^{+} \otimes \mathbb{Q}$ with a simple description: it is the ring generated over $\mathbb{Z}$ by the values $\phi_{0}(\mathfrak{a})+\phi_{0}(\overline{\mathfrak{a}})$ as $\mathfrak{a}$ runs over integral ideals of $\mathcal{O}_{K}$. For simplicity, let us assume $\mathcal{R}^{+}=\mathcal{O}_{T^{+}}$; our computations indicate that this is often the case, and, in general, $\mathcal{R}^{+}$is maximal locally at primes away from $h$. (We are not aware, however, of a general criterion guaranteeing the maximality of $\mathcal{R}^{+}$.) This ring acts on $\coprod_{B / \mathbb{Q}}$, and we are interested in the order ideal, or characteristic ideal, $\mathfrak{s}_{B}$, of this $\mathcal{R}^{+}$-module. Following Buhler-Gross [BG], we have the following conjecture.

Conjecture 3 (Refined Birch-Swinnerton-Dyer Conjecture). For $a$ prime $p>3$ satisfying $p \equiv 3 \bmod 8$, and $B=B(p)^{-3}, \mathfrak{s}_{B}=\mathfrak{f}^{2}$.

Since the norm of the order ideal is the cardinality of the module, in order to check that this conjecture is consistent with that of Birch and Swinnerton-Dyer, we must verify that the latter predicts the order of $\coprod_{B / \mathbb{Q}}$ to be $\mathbb{N}_{T^{+} / \mathbb{Q}}(\mathfrak{f})^{2}$. This is our main result (Theorem 5), and is proved below.

We follow Manin's notation and setup for the Birch-Swinnerton-Dyer conjecture [Ma]. For instance, for a place $v$ of $H^{+}$, we denote the local factor $\left|A\left(H_{v}^{+}\right) / A\left(H_{v}^{+}\right)^{0}\right|$ by $m_{v}$. The primes of bad reduction for $A / H^{+}$are the primes above $p$ and those above 3 ; for all other finite places $v, m_{v}=1$.

Lemma 4. With $\kappa$ as defined in (1), the local factors $m_{v}$ (for $v$ a place of $\mathrm{H}^{+}$) satisfy

i) $\prod_{v \mid p} m_{v}=2^{h}$

ii) $\prod_{v \mid 3} m_{v}=\kappa^{h}$;

iii) $\prod_{v \mid \infty} m_{v}=2^{-(h-1) / 2}\left(2 \pi\left|\eta\left(z_{\mathcal{O}}\right)\right|^{2} 3^{-1 / 2} p^{-1 / 4}\right)^{h} \prod_{C \in \mathrm{Cl}_{K}} u_{C}^{2}$. 
Proof. For $i$ ) and iii), we refer to the calculation of the same quantities for $A(p)$ in [RV, pp. 568-570], as they require little or no modification for $A(p)^{-3}$. For ii), we note that $\left(\prod_{v \mid 3} m_{v}\right)^{2}=\prod_{w \mid 3}\left|B\left(K_{w}\right) / B\left(K_{w}\right)^{0}\right|$, where $w$ runs over the primes of $K$ dividing 3 and $B=\operatorname{Res}_{H^{+} / \mathbb{Q}} A$, then use the formula on p. 232 of Gross [Gr2].

Theorem 5. The group of rational points $A\left(H^{+}\right)$is trivial. If the conjecture of Birch and Swinnerton-Dyer on the value of $L(1, A / F)$ holds, the cardinality of $\coprod_{A / H^{+}}$is $\mathbb{N}_{T^{+} / \mathbb{Q}}(\mathfrak{f})^{2}$, where $\mathfrak{f}$ is the integral $\mathcal{R}^{+}$-ideal defined in section 2.

Proof. By Theorem 2, and the factorization (4), $L\left(1, A / H^{+}\right) \neq 0$ since $s^{\varphi} \neq$ 0 for all $\varphi \in \widehat{\mathrm{Cl}_{K}}$. A theorem of Arthaud [Ar] and Rubin [Ru2], extending the Coates-Wiles theorem $[\mathrm{CW}]$, implies that $A / H^{+}$has rank 0. By Gross [Gr1], $A\left(H^{+}\right)$is torsion-free, hence trivial. Our base field $H^{+}$has $r_{2}\left(H^{+}\right)=$ $(h-1) / 2$ many pairs of complex embeddings and discriminant $\operatorname{disc}\left(H^{+} / \mathbb{Q}\right)=$ $p^{(h-1) / 2}$. When we combine Theorem 2 with Lemma 4 and compare with the Birch-Swinnerton-Dyer conjecture

$$
L(1, A / F) \stackrel{?}{=} \frac{\left|\coprod_{A / F}\right|}{\left|A(F)_{\text {tor }}\right|^{2}} \frac{2^{r_{2}(F)}}{|\operatorname{disc}(F / \mathbb{Q})|^{1 / 2}} \prod_{v} m_{v},
$$

we find that the predicted order of $\coprod_{A / H^{+}}$is $S^{2}$ where

$$
S=\prod_{\varphi \in \widehat{\mathrm{Cl}}_{K}} s^{\varphi} \prod_{C \in \mathrm{Cl}_{K}} u_{C}^{-1} .
$$

Using $\mathbb{N}_{T^{+} / \mathbb{Q}}(\mathfrak{f})^{h}= \pm \mathbb{N}_{M^{+} / \mathbb{Q}}(s)$, it is easily shown ([H, Lemma 2.7.v] $]$ that $S$ is a generator of the $\mathbb{Z}$-ideal $\prod_{\sigma \in \mathrm{Cl}_{K}} \mathfrak{f}^{\sigma}$, i.e. $S= \pm \mathbb{N}_{T^{+} / \mathbb{Q}}(\mathfrak{f}) \in \mathbb{Z}$, completing the proof.

\section{The class of the order ideal, and a question of Gross}

We continue to assume that $d=p>3$ is a prime satisfying $p \equiv 3 \bmod 8$. Let us return to the cocycle $w$ defined in section 3 . It is easily seen that the cohomology class $[w]^{h}$ is trivial, split by $\prod_{\sigma} w_{\sigma}^{-1}$, and it is natural to ask whether $[w]$ itself is trivial in $H^{1}\left(\operatorname{Gal}\left(M / T^{+}\right), E_{M}\right)$. Indeed, Gross [Gr3] constructed units (called $u_{\sigma}$ in $[\mathrm{H}]$ ) which are essentially the squares of $w_{\sigma}$ and asked the same question about their cohomology class $[u]$. The classes $[u]$ and $[w]$ in fact are either both trivial or both non-trivial; for more details, see $[\mathrm{H}]$, especially Theorem 3.4 and Questions 2.9, 2.10, 2.13.

Question 2.10 was answered in the negative in $[\mathrm{H}]$ by calculating some examples; here we will do the same for Questions 2.9 and 2.13 of $[\mathrm{H}]$. Before doing so, we recall ([H, Theorem 2.12]) that the cohomology class of $w$ is trivial if and only if the ideal class of $\mathfrak{f}$ in $\mathrm{Cl}_{T^{+}}$is trivial. Hence, assuming the above refined Birch-Swinnerton-Dyer conjecture, Gross' original question boils down to whether the ideal class of the Tate-Shafarevich order ideal of $B / \mathbb{Q}$ is trivial in 
the class group of $\mathcal{R}^{+}$. The difficulty in finding non-trivial examples is that the class number of $T^{+}$is seldom greater than 1 and very seldom has a non-trivial factor in common with $h$, which is what we need since [f] is killed by $h$. In one case previously investigated where $h_{T^{+}}$and $h$ have a factor in common, namely $p=4027$, it turned out that $\mathfrak{f}$ was principal $[\mathrm{H}]$. Here we present three examples where the predicted order ideal of $\coprod_{B / \mathbb{Q}}$, i.e. $\mathfrak{f}^{2}$, is not principal in $\mathcal{R}^{+}$, though of course it capitulates in $M^{+}$. Let us write $|\coprod|$ ? for the order of $\coprod_{B / \mathbb{Q}}$ as predicted by Birch-Swinnerton-Dyer, namely $S^{2}$ where $S$ is given by (5).

Example 1. $p=571, h=5,|\amalg \amalg|_{?}=4^{2}, \mathfrak{f}=\mathfrak{p}_{2}^{2}$, where $\mathfrak{p}_{2}$ is the unique prime of degree 1 above 2. Here, $\mathfrak{p}_{2}$ generates the ideal class group of $\mathcal{R}^{+}=\mathcal{O}_{T^{+}}$(it has order 5), hence $\mathfrak{f}$ and $\mathfrak{f}^{2}$ are not principal. A defining polynomial for $T^{+}$is $x^{5}-24 x^{3}+125 x-58$.

Example 2. $p=1523, h=7,|\amalg \amalg|_{?}=2485^{2}, \mathfrak{f}=\mathfrak{p}_{5} \mathfrak{p}_{7} \mathfrak{p}_{71}$; as before, $\mathfrak{p}_{r}$ is the unique prime of degree 1 above $r$. The class group of $\mathcal{R}^{+}=\mathcal{O}_{T^{+}}$has order 7 and $\mathfrak{f}$ generates it. We have $\mathcal{R}^{+} \cong \mathbb{Z}[\theta]$ where $\theta$ is a root of $x^{7}-21 x^{5}+126 x^{3}-189 x+85$.

Example 3. $p=3019, h=7,|\amalg|_{\text {? }}=15373^{2}$. The prime 15373 splits into 7 prime ideals in $\mathcal{R}^{+}=\mathcal{O}_{T^{+}}$, one of which is $\mathfrak{f}$. All seven of these primes give rise to the same non-trivial class in the class group of $\mathcal{R}^{+}$, which has order 7 . If the predicted order is in fact correct, then $\amalg \cong\left(\mathcal{R}^{+} / \mathfrak{f}\right)^{2}$ as $\mathcal{R}^{+}$-modules. A defining polynomial for $T^{+}$is $x^{7}-35 x^{5}+350 x^{3}-875 x+514$.

Remarks. 1) These calculations were carried out in GP/Pari [B] on a Power Computing Power 100. We double-checked the calculation of $L\left(1, A / H^{+}\right)$by using the standard algorithm [BG] as well.

2) In examples 1 and 3 , the class of the different in $\mathcal{R}^{+}$is not principal, so there is no power basis for $\mathcal{R}^{+} / \mathbb{Z}$. According to de Smit [dS], the non-triviality of the class of the different implies that, as $\mathbb{Z}$-algebra, $\mathcal{R}^{+}$is not a complete intersection. Note that $\mathcal{R}^{+}$is a quotient of the Hecke algebra $\mathbb{T} \subset \operatorname{End}\left(J_{0}\left(9 p^{2}\right)\right)$.

\section{Calculating $L(1, \psi)$}

We now give a proof of Theorem 2. We divide this section into three parts. In the first two subsections, we relax the condition on the discriminant $-d$, requiring only that it be prime to 6 . In the third subsection, we return to the case where $d=p>3$ is a prime $\equiv 3 \bmod 8$, and complete the proof of Theorem 2 .

5.1. Eisenstein series of weight 1 . For an ideal $\mathfrak{a}$ of $\mathcal{O}_{K}$ prime to $6 d$, define a partial Hecke series

$$
Z(s, \mathfrak{a})=\frac{1}{2} \sum_{\lambda \in \mathfrak{a}}^{\prime} \frac{\epsilon(\lambda) \chi\left(|\lambda|^{2}\right) \bar{\lambda}}{|\lambda|^{2 s}}, \quad(\Re(s)>3 / 2) .
$$

As usual, the prime indicates that the sum is over the non-zero elements of $\mathfrak{a}$. Via a standard argument, one expresses the Hecke $L$-series $L(s, \psi)=$ 
$\sum_{\mathfrak{b} \subseteq \mathcal{O}_{K}} \psi(\mathfrak{b}) \mathbb{N b}^{-s}$ in terms of the $Z(s, \mathfrak{a})$ :

$$
L(s, \psi)=\sum_{[\mathfrak{a}] \in \mathrm{Cl}_{K}} \frac{\psi(\mathfrak{a})}{\mathbb{N a}^{1-s}} Z(s, \mathfrak{a}) .
$$

Recall the Eisenstein series (of weight 1 and character $\epsilon$ on $\Gamma_{0}(d)$ )

$$
G_{1, \epsilon}(z)=\frac{1}{2} \sum_{m, n \in \mathbb{Z}}^{\prime} \frac{\epsilon(n)}{m d z+n} \quad(z \in \mathcal{H}),
$$

which is not an absolutely convergent series, but is summed by the Hecke trick

$$
G_{1, \epsilon}(z)=\left.\frac{1}{2} \sum_{m, n}^{\prime} \frac{\epsilon(n)}{m d z+n} \frac{1}{|m d z+n|^{2 s}}\right|_{s=0} .
$$

Its Fourier expansion is given by Hecke [He, Werke p. 454]

$$
G_{1, \epsilon}(z)=L(1, \epsilon)+\frac{2 \pi}{\sqrt{d}} \sum_{n \geq 1} r_{n} e^{2 \pi i n z}
$$

where $r_{n}=\sum_{m \mid n} \epsilon(m)$ is the number of ideals of norm $n$ in $\mathcal{O}_{K}$. For an ideal $\mathfrak{a}$ of $\mathcal{O}_{K}$, the associated binary theta series

$$
\Theta_{\mathfrak{a}}(z)=\frac{1}{2} \sum_{\mathfrak{b} \subseteq \mathcal{O}_{K},[\mathfrak{b}]=[\mathfrak{a}]} e^{2 \pi i z \mathbb{N} \mathfrak{b}} \quad(z \in \mathcal{H}),
$$

is a weight-one modular form (on $\Gamma_{0}(d)$ with character $\epsilon$ ) which depends only on the class $[\mathfrak{a}] \in \mathrm{Cl}_{K}$. By (8) and Dirichlet's class number formula $(L(1, \epsilon)=$ $\pi h / \sqrt{d})$, the $h$ binary series of discriminant $-d$ add up to a constant times $G_{1, \epsilon}$ :

$$
G_{1, \epsilon}(z)=\frac{2 \pi}{\sqrt{d}} \sum_{[\mathfrak{a}] \in \mathrm{Cl}_{K}} \Theta_{\mathfrak{a}}(z)
$$

In [RVZ], $L(1, \phi)$ was expressed as a sum of values of $G_{1, \epsilon}$ at CM-points. Here we do the same for $L(1, \psi)$, but the expression is, at least initially, more complicated as we must pass through an intermediary twisted Eisenstein series

$$
G_{1, \epsilon, \chi}(z)=\frac{1}{2} \sum_{m, n}^{\prime} \frac{\epsilon(n) \chi\left(m^{2} d+n^{2}\right)}{m d z+n},
$$

again summed via Hecke's trick. One way to calculate the Fourier expansion of $G_{1, \epsilon, \chi}$ is to relate it to $G_{1, \epsilon}$.

Lemma 6. For all $z \in \mathcal{H}, G_{1, \epsilon, \chi}(z)=\kappa\left(G_{1, \epsilon}(3 z)-\frac{1}{3} G_{1, \epsilon}(z / 3)\right)$. 
Proof. We can break up the sum (7) according to congruence classes modulo 3, and verify:

$$
\begin{gathered}
\left.\frac{1}{2} \sum_{\substack{m \in 3 \mathbb{Z} \\
n \notin 3 \mathbb{Z}}} \frac{\epsilon(n)}{m d z+n} \frac{1}{|m d z+n|^{2 s}}\right|_{s=0}=G_{1, \epsilon}(3 z)-\frac{\epsilon(3)}{3} G_{1, \epsilon}(z), \\
\left.\chi(d) \frac{1}{2} \sum_{\substack{m \notin 3 \mathbb{Z} \\
n \in 3 \mathbb{Z}}} \frac{\epsilon(n)}{m d z+n} \frac{1}{|m d z+n|^{2 s}}\right|_{s=0}= \\
\left.\chi(d+1) \frac{1}{2} \sum_{\substack{m \notin 3 \mathbb{Z} \\
n \notin 3 \mathbb{Z}}} \frac{\epsilon(n) \frac{\epsilon(3)}{m d z+n}\left(G_{1, \epsilon}\left(\frac{z}{3}\right)-G_{1, \epsilon}(z)\right),}{|m d z+n|^{2 s}}\right|_{s=0}= \\
\chi(d+1)\left[\left(1+\frac{\epsilon(3)}{3}\right) G_{1, \epsilon}(z)-G_{1, \epsilon}(3 z)-\frac{\epsilon(3)}{3} G_{1, \epsilon}\left(\frac{z}{3}\right)\right] .
\end{gathered}
$$

Adding these together, and taking note of the identities $\chi(d)=-\epsilon(3), \chi(d+1)=$ $(\epsilon(3)-1) / 2$, we get the desired formula.

We are now ready to express the critical value $L(1, \psi)$ in terms of binary theta series evaluated at CM points.

Lemma 7. With $\kappa$ as in (1), we have

$$
L(1, \psi)=\frac{2 \pi \kappa}{\sqrt{d}} \sum_{[\mathfrak{a}],\left[\mathfrak{a}_{1}\right] \in \mathrm{Cl}_{K}} \bar{\psi}(\mathfrak{a})^{-1}\left(\Theta_{\mathfrak{a} \mathfrak{a}_{1}}\left(3 \tau_{\mathfrak{a}}\right)-\frac{1}{3} \Theta_{\mathfrak{a} \mathfrak{a}_{1}}\left(\frac{\tau_{\mathfrak{a}}}{3}\right)\right) .
$$

Proof. Suppose $\mathfrak{a}$ is primitive and has norm $a$ prime to $6 d$. Elements $\lambda \in \mathfrak{a}$ correspond to integer pairs $m, n$ via $\lambda=a\left(m d \tau_{\mathfrak{a}}+n\right)$; for this $\lambda$, one easily checks that $\epsilon(\lambda)=\epsilon(n), \chi\left(|\lambda|^{2}\right)=\chi\left(m^{2} d+n^{2}\right)$. Hence

$$
Z(s, \mathfrak{a})=\frac{a^{1-2 s}}{2} \sum_{m, n}^{\prime} \frac{\epsilon(n) \chi\left(m^{2} d+n^{2}\right)\left(\overline{m d \tau_{\mathfrak{a}}+n}\right)}{\left|m d \tau_{\mathfrak{a}}+n\right|^{2 s}}, \quad(\Re(s)>3 / 2) .
$$

In particular,

$$
Z(1, \mathfrak{a})=a^{-1} G_{1, \epsilon, \chi}\left(\tau_{\mathfrak{a}}\right)
$$

Combining (10) with (2) and (6), and using Lemma 6 together with (9), we arrive at the desired formula. 
5.2. A factorization formula. Our goal in this subsection is to express each term in the sum appearing in Lemma 7 as a product of two $\eta$-values times simple constants. We derive this from a suitable generalization of the factorization formula of Rodriguez Villegas and Zagier [RVZ], which reads

$$
\begin{aligned}
\sum_{m, n \in \mathbb{Z}} e^{2 \pi i(m \nu+n \mu)} e^{\pi\left(i m n-Q_{z}(m, n)\right) / a} & = \\
& \sqrt{2 a y} \theta\left[\begin{array}{c}
a \mu \\
\nu
\end{array}\right](z / a) \theta\left[\begin{array}{c}
\mu \\
-a \nu
\end{array}\right](-a \bar{z}),
\end{aligned}
$$

where $a$ is a positive integer, $z=x+i y \in \mathcal{H}, Q_{z}(m, n)=|m z-n|^{2} / 2 y$ is a quadratic form of discriminant -1 , and the theta function $\theta\left[\begin{array}{l}\mu \\ \nu\end{array}\right]$ with arbitrary characteristics $\mu, \nu \in \mathbb{Q}$ is defined by

$$
\theta\left[\begin{array}{l}
\mu \\
\nu
\end{array}\right](z)=\sum_{n \in \mathbb{Z}} e^{\pi i(n+\mu)^{2} z+2 \pi i \nu(n+\mu)} .
$$

More generally, for any function $f$ on $\mathbb{Z}$, we put

$$
\theta_{f}\left[\begin{array}{l}
\mu \\
\nu
\end{array}\right](z)=\sum_{n \in \mathbb{Z}} f(n) e^{\pi i(n+\mu)^{2} z+2 \pi i \nu(n+\mu)} .
$$

For later reference, we note the identities

$$
\theta\left[\begin{array}{c}
\mu+r \\
\nu
\end{array}\right](z)=\theta\left[\begin{array}{c}
\mu \\
\nu
\end{array}\right](z), \quad \theta\left[\begin{array}{c}
\mu \\
\nu+r
\end{array}\right](z)=e^{2 \pi i \mu r} \theta\left[\begin{array}{c}
\mu \\
\nu
\end{array}\right](z), \quad(r \in \mathbb{Z})
$$

For a positive integer $N$, and a function $g$ modulo $N$, recall that the (finite) Fourier Transform $\hat{g}$ of $g$ is defined by

$$
\hat{g}(s)=\sum_{r \bmod N} g(r) e_{N}(-r s),
$$

where $e_{N}(x)=e^{2 \pi i x / N}$. For an integer $a$ relatively prime to $N$, and functions $f, g$ modulo $N$, we introduce the (finite) Wigner Transform $W_{f, g}^{(a)}$ defined (as a function of pairs of integers modulo $N$ ) by

$$
W_{f, g}^{(a)}(m, n)=\sum_{r, s \bmod N} f(r) \hat{g}(s) e_{N}(a r s) e_{N}(m s+n r) .
$$

(For the classical Wigner transform, see Folland [Fo]). A simple change of variables yields the expression

$$
W_{f, g}^{(a)}(m, n)=N \sum_{r \bmod N} f(r) g(a r+m) e_{N}(r n) .
$$

We are now ready to state the promised factorization formula. 
Proposition 8. Suppose $a, N$ are relatively prime positive odd integers, and $\mu, \nu$ are rational numbers; assume that the denominator of $\mu$ is relatively prime to $N$. For functions $f, g$ modulo $N$, and $z=x+i y$ in the upper half-plane, we have

$$
\begin{gathered}
\sum_{m, n \in \mathbb{Z}} e^{2 \pi i(m \nu+n \mu)} W_{f, g}^{(a)}(m, n) e^{\pi\left(i m n-Q_{z}(m, n)\right) / a N}= \\
N \sqrt{2 y a N} \theta_{g}\left[\begin{array}{c}
a N \mu \\
\nu
\end{array}\right](z / a N) \theta_{f}\left[\begin{array}{c}
N \mu \\
-a \nu
\end{array}\right](-a \bar{z} / N) .
\end{gathered}
$$

Proof. Suppose $r, s$ are integers and that $s \mu \in \mathbb{Z}$. We consider the factorization formula (11) with new parameters $\mu+r / N, \nu+s / N, a N$ in place of $\mu, \nu, a$, and multiply both sides by $f(r) \hat{g}(s) e_{N}($ ars $)$, then add over $r, s$ modulo $N$ to get

$$
\begin{aligned}
& \sum_{m, n \in \mathbb{Z}} e^{2 \pi i(m \nu+n \mu)} W_{f, g}^{(a)}(m, n) e^{\pi\left(i m n-Q_{z}(m, n)\right) / a N}= \\
& \sqrt{2 y a N} \sum_{s \bmod N} \hat{g}(s) \theta\left[\begin{array}{c}
a N \mu \\
\nu+s / N
\end{array}\right](z / a N) \sum_{r \bmod N} f(r) \theta\left[\begin{array}{c}
\mu+r / N \\
-a N \nu
\end{array}\right](-a N \bar{z}) .
\end{aligned}
$$

In the first sum on the right hand side of the above equation, we may choose representatives $s \bmod N$ with $s \mu \in \mathbb{Z}$ since $N$ is prime to the denominator of $\mu$. It remains to simplify the two sums of theta series. First, the sum over $s$ :

$$
\begin{aligned}
\sum_{s \bmod N} \hat{g}(s) \theta\left[\begin{array}{c}
a N \mu \\
\nu+s / N
\end{array}\right](z / a N) & =\sum_{m \in \mathbb{Z}+a N \mu} \sum_{s \bmod N} \hat{g}(s) e_{N}(s m) e^{2 \pi i \nu m} e^{\frac{2 \pi i m^{2} z}{a N}}, \\
& =\sum_{n \in \mathbb{Z}} N g(n) e^{2 \pi i \nu(n+a N \mu)} e^{\frac{\pi i(n+a N \mu)^{2} z}{a N}} \\
& =N \theta_{g}\left[\begin{array}{c}
a N \mu \\
\nu
\end{array}\right](z / a N) .
\end{aligned}
$$

For the sum over $r$, we write:

$$
\sum_{r \bmod N} f(r) \theta\left[\begin{array}{c}
\mu+r / N \\
-a N \nu
\end{array}\right](-a N \bar{z})=\sum_{m \in \mathbb{Z}+\mu+r / N} f(r) e^{-2 \pi i m a N \nu} e^{-\pi i m^{2} a N \bar{z}},
$$

then make a change of variables

$$
m=\mu+n / N, \quad n \in \mathbb{Z}, n \equiv r \bmod N, \quad m^{2}=\frac{(n+N \mu)^{2}}{N^{2}},
$$

to get

$$
\begin{aligned}
& =\sum_{r \bmod N} \sum_{n \equiv r \bmod N} f(n) e^{2 \pi i(-a \nu)(n+N \mu)} e^{\pi i(n+N \mu)^{2}(-a \bar{z} / N)} \\
& =\theta_{f}\left[\begin{array}{c}
N \mu \\
-a \nu
\end{array}\right](-a \bar{z} / N) .
\end{aligned}
$$

This completes the proof. 
Proposition 9. For ideals $\mathfrak{a}, \overline{\mathfrak{a}}_{1}$ of $\mathcal{O}_{K}$ relatively prime to $6 d$ and to each other,

$$
\Theta_{\mathfrak{a} \mathfrak{a}_{1}}\left(3 \tau_{\mathfrak{a}}\right)-\frac{1}{3} \Theta_{\mathfrak{a a}_{1}}\left(\tau_{\mathfrak{a}} / 3\right)=\frac{2 \delta d^{1 / 4}}{\sqrt{3 a_{1}}}\left(\frac{3}{a}\right) \eta\left(z_{\mathfrak{a}^{2} \mathfrak{a}_{1}}\right) \overline{\eta\left(z_{\mathfrak{a}_{1}}\right)} .
$$

Proof. We first plug in $\mu=1 / 2, \nu=\delta / 2, f=g=\chi$ into the factorization formula and use the identity

$$
\theta_{\chi}\left[\begin{array}{c}
3 r / 2 \\
s / 2
\end{array}\right](z / 3)=2 \delta e^{3 \pi i r s / 4}\left(\frac{-4}{r}\right) \eta(z), \quad(r \equiv 1 \bmod 2, s \equiv \delta \bmod 2, z \in \mathcal{H}),
$$

which is easily verified using (3), to obtain

$$
\begin{aligned}
\sum_{m, n \in \mathbb{Z}}(-1)^{m \delta+n} W_{\chi, \chi}^{(a)}(m, n) e^{\pi\left(i m n-Q_{z}(m, n)\right) / 3 a} & = \\
& 12 \delta \sqrt{6 y a}\left(\frac{-4}{a}\right) \eta(z / a) \overline{\eta(a z)} .
\end{aligned}
$$

Recall that for $z=x+i y, Q_{z}(m, n)=|m z-n|^{2} / 2 y$. We may choose a basis

$$
\mathfrak{a}^{2} \mathfrak{a}_{1}=\left[a^{2} a_{1},(b+\sqrt{-d}) / 2\right], \quad b \in 3 d \mathbb{Z}, \quad b \equiv 1 \bmod 16,
$$

and put

$$
z=\frac{b+\sqrt{-d}}{2 a a_{1}}
$$

so that $a_{1} z / d=\tau_{\mathfrak{a}}, z / a=z_{\mathfrak{a}^{2} \mathfrak{a}_{1}}$, and $a z=z_{\mathfrak{a}_{1}}$. The integer $c=\left(b^{2}+d\right) / 4 a a_{1}$ is odd and divisible by $a$. To simplify the notation, let

$$
Q(m, n)=Q_{z}(m, n) / \sqrt{d}=c m^{2}-b m n+a a_{1} n^{2}
$$

be a quadratic form associated to $\mathfrak{a a}_{1}=a a_{1}[1, z]$. Substituting the congruence

$$
\pi\left(i m n-Q_{z}(m, n)\right) / 3 a \equiv 2 \pi i\left(Q(m, n) \tau_{\mathfrak{a}} / 3+\frac{m \delta+n}{2}-a m n / 3\right) \bmod 2 \pi i \mathbb{Z}
$$

into (16), we find

$$
\begin{aligned}
\sum_{m, n \in \mathbb{Z}} W_{\chi, \chi}^{(a)}(m, n) e_{3}(-a m n) e^{2 \pi i Q(m, n) \tau_{\mathfrak{a}} / 3} & = \\
& 12 \delta d^{1 / 4} \sqrt{\frac{3}{a_{1}}}\left(\frac{-4}{a}\right) \eta\left(z_{\mathfrak{a}^{2} \mathfrak{a}_{1}}\right) \overline{\eta\left(z_{\mathfrak{a}_{1}}\right)} .
\end{aligned}
$$

It is easy (using (13), say) to verify that

$$
\frac{1}{3} W_{\chi, \chi}^{(a)}(m, n) e_{3}(-a m n)= \begin{cases}2 \chi(a) & \text { if } m \equiv n \equiv 0 \bmod 3 \\ -\chi(a) & \text { otherwise }\end{cases}
$$


Plugging this into the previous equation yields the result since

$$
\Theta_{\mathfrak{a} \mathfrak{a}_{1}}\left(3 \tau_{\mathfrak{a}}\right)=\frac{1}{2} \sum_{m, n \in 3 \mathbb{Z}} e^{2 \pi i Q(m, n) \tau_{\mathfrak{a}} / 3} .
$$

From the transformation properties of the modular form $\Theta_{\mathfrak{a}}(z)$ under homotheties of $\mathfrak{a}$ and under the $\Gamma_{0}(d)$-action on $z$, we deduce the following:

Corollary 10. Suppose $\mathfrak{a}, \overline{\mathfrak{a}}_{1}$ are as in the Proposition, and that $d \equiv 3 \bmod 8$. Then,

$$
\left(\frac{-4}{\mathbb{N a}}\right) \bar{\phi}(\mathfrak{a})^{-1} \eta\left(z_{\mathfrak{a}^{2} \mathfrak{a}_{1}}\right)
$$

depends only the Hecke character $\phi$, the ideal $\mathfrak{a}_{1}$ and the ideal class $[\mathfrak{a}]$ of $\mathfrak{a}$.

When $d \equiv 7(\bmod 8)$, i.e. $\delta=0$, we see from Proposition 9 , together with (10) and (9), that each $Z(1, \mathfrak{a})$ vanishes, in particular $L(1, \psi)=0$. Of course, the latter also follows easily from the calculation of the sign in the functional equation of $L(s, \psi)$ [Gr1, Theorem 19.1.1].

5.3. Proof of Theorem 2. Suppose $d=p>3$ is $\equiv 3 \bmod 8$. We are finally ready to write $L(1, \psi)$ as a period times the square of a non-zero algebraic integer in $M^{+}$.

Proof of Theorem 2. Combining Proposition 9 and Lemma 7, we have

$$
L\left(1, \psi_{0} \varphi\right)=\frac{4 \pi \kappa}{3^{1 / 2} p^{1 / 4}} \sum_{[\mathfrak{a}],\left[\mathfrak{a}_{1}\right]} \frac{\left(\overline{\varphi \psi_{0}}\right)(\mathfrak{a})^{-1}}{\sqrt{a_{1}}}\left(\frac{3}{a}\right) \eta\left(z_{\mathfrak{a}^{2} \mathfrak{a}_{1}}\right) \overline{\eta\left(z_{\mathfrak{a}_{1}}\right)} .
$$

Since $h$ is odd, every ideal class is representable by a square ideal. Now we change variables twice, first replacing $\mathfrak{a}_{1}$ by $\mathfrak{a}_{1}^{2}$, then replacing $\mathfrak{a}$ by $\mathfrak{a a}_{1}^{-1}$ to get

$$
\begin{aligned}
L\left(1, \psi_{0} \varphi\right) & =\frac{4 \pi \kappa}{3^{1 / 2} p^{1 / 4}} \sum_{[\mathfrak{a}],\left[\mathfrak{a}_{1}\right]}\left(\overline{\varphi \psi_{0}}\right)(\mathfrak{a})^{-1}\left(\frac{3}{a}\right) \eta\left(z_{\mathfrak{a}^{2}}\right) \frac{\left(\overline{\varphi \psi_{0}}\right)\left(\mathfrak{a}_{1}\right)}{a_{1}}\left(\frac{3}{a_{1}}\right) \overline{\eta\left(z_{\mathfrak{a}_{1}^{2}}\right)} \\
& =\frac{4 \pi \kappa}{3^{1 / 2} p^{1 / 4}}\left|\sum_{[\mathfrak{a}]}\left(\overline{\varphi \psi_{0}}\right)(\mathfrak{a})^{-1}\left(\frac{-4}{\overline{N a}}\right) \eta\left(z_{\mathfrak{a}^{2}}\right)\right|^{2} \\
& =\frac{4 \pi \kappa\left|\eta\left(z_{\mathcal{O}}\right)\right|^{2}}{3^{1 / 2} p^{1 / 4}}\left|\sum_{C \in \mathrm{Cl}_{K}} u_{C}^{\varphi}\right|^{2} .
\end{aligned}
$$

This completes the proof, since $s^{\varphi}$ is real.

Corollary 11. For $\psi \in \Psi, L(1, \psi)>0$. 


\section{Tables}

In this section, we present the results of numerical calculations in the form of a few tables. In the first table, for primes $3<p<3000$, congruent to 3 modulo 8 , we list $p, h$, and $S= \pm \mathbb{N}_{T^{+} / \mathbb{Q}}(\mathfrak{f})$ (computed via (5)), the square root of the predicted order of $\coprod_{B / \mathbb{Q}}$. Since $u_{C}$ is a unit, it is immediate from our formula that $A(p)^{-3}$ has trivial predicted Tate-Shafarevich group for the class number one discriminants $-p=-11,-19,-43,-67,-163$. Of the 108 cases listed in table 1, 44 have even $S, 39$ have $S$ divisible by 3 , and 20 have $S$ divisible by 5 . None has $S$ divisible by $p$.

In the second, third and fourth tables, for primes $p \equiv 3 \bmod 8$ such that $\mathbb{Q}(\sqrt{-p})$ has class number $h=3,5$, or 7 , and such that $\mathcal{R}^{+}$is the maximal order $\mathcal{O}_{T^{+}}$, we list $p$ and $\mathfrak{f}$, the ideal whose square is conjecturally the order ideal of the Tate-Shafarevich group of $B / \mathbb{Q}$. In all cases other than the three detailed in section 4 , the $\mathcal{R}^{+}$-ideal $\mathfrak{f}$ is principal. The notation for these tables is as follows: $\mathfrak{p}_{r}$ denotes a prime of degree one over the rational prime $r$, and and $\mathfrak{q}_{r, f}$ denotes a prime of degree $f>1$ over $r$. When there is more than one prime of degree 1 , respectively of degree $f>1$, in $\mathcal{R}^{+}$, we write instead $\mathfrak{p}_{r}^{\prime}, \mathfrak{p}_{r}^{\prime \prime}, \ldots$, respectively $\mathfrak{q}_{r, f}^{\prime}, \mathfrak{q}_{r, f}^{\prime \prime}, \ldots$ In the latter cases, we have not specified exactly which ideals occur in $\mathfrak{f}$, in order to keep the notation from becoming even more cumbersome. Note that primes of degree one are much more prevalent.

Finally, we take this opportunity to make a few remarks on a table (for the curve $A(p), p \equiv 7 \bmod 8)$, which appears in $[\mathrm{RV}]$; it was noted in that paper that within the range of calculations $(p<3000)$ the number $\mathcal{S}(p)$ (whose square is the predicted order of $\coprod_{A(p) / F}$ ) was rarely even, and never divisible exactly by 2. The latter part of this observation is easily explained: it was shown by Gross [Gr1] that $\operatorname{Gal}(H / K)$, a group of odd order, acts non-trivially (indeed without fixed points) on the 2-part of $\coprod_{A(p) / F}$, and this action commutes with the Cassels-Tate pairing. It follows (e.g. from Iwasawa $[\mathrm{Iw}]$ ) that the 2-rank of $\coprod_{A(p) / F}$, if non-zero, is at least $2 f$ where $f$ is the minimum, over prime divisors $q$ of $h$, of the order of 2 in $(\mathbb{Z} / q \mathbb{Z})^{*}$. As for the rarity of $\coprod_{A(p) / F}$ of even order, Gross has pointed out that there is heuristic evidence (and we have verified numerically) that the 2-part of this group is trivial if and only if the 2-part of the class group of $F$ is trivial. The Cohen-Lenstra heuristic suggests that the latter should often be the case (again sustained by numerical data [Ha]) since the class group of $F$ is non-cyclic whenever it is not trivial.

\begin{tabular}{|c|c|r|r|r|r|r|r|r|r|r|r|}
\hline$p$ & $h$ & $S$ & $|S|$ & $p$ & $h$ & $S$ & $|S|$ & $p$ & $h$ & $S$ & $|S|$ \\
\hline 11 & 1 & 1 & 1 & 131 & 5 & -6 & $2 \cdot 3$ & 283 & 3 & -11 & 11 \\
19 & 1 & 1 & 1 & 139 & 3 & -1 & 1 & 307 & 3 & -8 & $2^{3}$ \\
43 & 1 & 1 & 1 & 163 & 1 & 1 & 1 & 331 & 3 & -7 & 7 \\
59 & 3 & -3 & 3 & 179 & 5 & 20 & $2^{2} \cdot 5$ & 347 & 5 & -36 & $2^{2} \cdot 3^{2}$ \\
67 & 1 & 1 & 1 & 211 & 3 & 3 & 3 & 379 & 3 & -13 & 13 \\
83 & 3 & -5 & 5 & 227 & 5 & 8 & $2^{3}$ & 419 & 9 & 2143 & 2143 \\
107 & 3 & -1 & 1 & 251 & 7 & 358 & $2 \cdot 179$ & 443 & 5 & 100 & $2^{2} \cdot 5^{2}$ \\
\hline
\end{tabular}




\begin{tabular}{|c|c|c|c|}
\hline$p$ & $h$ & $S$ & $|S|$ \\
\hline 467 & 7 & 44 & $2^{2} \cdot 11$ \\
\hline 491 & 9 & -105 & $3 \cdot 5 \cdot 7$ \\
\hline 499 & 3 & -9 & $3^{2}$ \\
\hline 523 & 5 & 11 & 11 \\
\hline 547 & 3 & 12 & $2^{2} \cdot 3$ \\
\hline 563 & 9 & 381 & $3 \cdot 127$ \\
\hline 571 & 5 & -4 & $2^{2}$ \\
\hline 587 & 7 & 90 & $2 \cdot 3^{2} \cdot 5$ \\
\hline 619 & 5 & 15 & $3 \cdot 5$ \\
\hline 643 & 3 & 13 & 13 \\
\hline 659 & 11 & 473 & $11 \cdot 43$ \\
\hline 683 & 5 & -31 & 31 \\
\hline 691 & 5 & 19 & 19 \\
\hline 739 & 5 & 4 & $2^{2}$ \\
\hline 787 & 5 & -15 & $3 \cdot 5$ \\
\hline 811 & 7 & 246 & $2 \cdot 3 \cdot 41$ \\
\hline 827 & 7 & -152 & $2^{3} \cdot 19$ \\
\hline 859 & 7 & 289 & $17^{2}$ \\
\hline 883 & 3 & 7 & 7 \\
\hline 907 & 3 & 25 & $5^{2}$ \\
\hline 947 & 5 & -15 & $3 \cdot 5$ \\
\hline 971 & 15 & 702121 & $7^{3} \cdot 23 \cdot 89$ \\
\hline 1019 & 13 & -176 & $2^{4} \cdot 11$ \\
\hline 1051 & 5 & 89 & 89 \\
\hline 1091 & 17 & 311264513 & $7^{2} \cdot 67 \cdot 94811$ \\
\hline 1123 & 5 & 144 & $2^{4} \cdot 3^{2}$ \\
\hline 1163 & 7 & 166 & $2 \cdot 83$ \\
\hline 1171 & 7 & -23 & 23 \\
\hline 1187 & 9 & -9354 & $2 \cdot 3 \cdot 1559$ \\
\hline 1259 & 15 & 34101824 & $2^{6} \cdot 23 \cdot 23167$ \\
\hline 1283 & 11 & -207248 & $2^{4} \cdot 12953$ \\
\hline 1291 & 9 & -187 & $11 \cdot 17$ \\
\hline 1307 & 11 & 1739184 & $2^{4} \cdot 3 \cdot 19 \cdot 1907$ \\
\hline 1427 & 15 & -26904385 & $5 \cdot 71 \cdot 75787$ \\
\hline 1451 & 13 & 761379 & $3 \cdot 17 \cdot 14929$ \\
\hline 1459 & 11 & -74928 & $2^{4} \cdot 3 \cdot 7 \cdot 223$ \\
\hline 1483 & 7 & 8 & $2^{3}$ \\
\hline 1499 & 13 & 45498 & $2 \cdot 3 \cdot 7583$ \\
\hline 1523 & 7 & 2485 & $5 \cdot 7 \cdot 71$ \\
\hline 1531 & 11 & -22720 & $2^{6} \cdot 5 \cdot 71$ \\
\hline 1571 & 17 & 242697813 & $3 \cdot 199 \cdot 223 \cdot 1823$ \\
\hline 1579 & 9 & 7611 & $3 \cdot 43 \cdot 59$ \\
\hline 1619 & 15 & -88275739 & $29 \cdot 401 \cdot 7591$ \\
\hline
\end{tabular}


ON THE TATE-SHAFAREVICH GROUP OF CERTAIN ELLIPTIC CURVES 653

\begin{tabular}{|c|c|c|c|}
\hline$p$ & $h$ & $\bar{S}$ & $|S|$ \\
\hline 1627 & 7 & 747 & $3^{2} \cdot 83$ \\
\hline 1667 & 13 & 9626039 & 9626039 \\
\hline 1699 & 11 & -33342 & $2 \cdot 3 \cdot 5557$ \\
\hline 1723 & 5 & -175 & $5^{2} \cdot 7$ \\
\hline 1747 & 5 & -190 & $2 \cdot 5 \cdot 19$ \\
\hline 1787 & 7 & -1942 & $2 \cdot 971$ \\
\hline 1811 & 23 & -27172020350 & $2 \cdot 5^{2} \cdot 43 \cdot 12638149$ \\
\hline 1867 & 5 & -609 & $3 \cdot 7 \cdot 29$ \\
\hline 1907 & 13 & 303885 & $3^{3} \cdot 5 \cdot 2251$ \\
\hline 1931 & 21 & -119115284992 & $2^{9} \cdot 11 \cdot 21149731$ \\
\hline 1979 & 23 & 640380659636 & $2^{2} \cdot 19 \cdot 491 \cdot 17161021$ \\
\hline 1987 & 7 & 2307 & $3 \cdot 769$ \\
\hline 2003 & 9 & -229719 & $3 \cdot 7 \cdot 10939$ \\
\hline 2011 & 7 & 54 & $2 \cdot 3^{3}$ \\
\hline 2027 & 11 & 144642 & $2 \cdot 3 \cdot 24107$ \\
\hline 2083 & 7 & -13409 & $11 \cdot 23 \cdot 53$ \\
\hline 2099 & 19 & 39574291187 & $83 \cdot 476798689$ \\
\hline 2131 & 13 & -4031 & $29 \cdot 139$ \\
\hline 2179 & 7 & -108 & $2^{2} \cdot 3^{3}$ \\
\hline 2203 & 5 & -286 & $2 \cdot 11 \cdot 13$ \\
\hline 2243 & 15 & -12460096 & $2^{6} \cdot 11^{2} \cdot 1609$ \\
\hline 2251 & 7 & 1008 & $2^{4} \cdot 3^{2} \cdot 7$ \\
\hline 2267 & 11 & 1598897 & 1598897 \\
\hline 2339 & 19 & -27980957102 & $2 \cdot 7 \cdot 523 \cdot 3821491$ \\
\hline 2347 & 5 & 635 & $5 \cdot 127$ \\
\hline 2371 & 13 & 90441 & $3^{2} \cdot 13 \cdot 773$ \\
\hline 2411 & 23 & -237374573222 & $2 \cdot 37 \cdot 1163 \cdot 2758181$ \\
\hline 2459 & 19 & 96436584 & $2^{3} \cdot 3^{2} \cdot 67 \cdot 19991$ \\
\hline 2467 & 7 & 72713 & $19 \cdot 43 \cdot 89$ \\
\hline 2531 & 17 & 20814832370 & $2 \cdot 5 \cdot 73 \cdot 547 \cdot 52127$ \\
\hline 2539 & 11 & 24685 & $5 \cdot 4937$ \\
\hline 2579 & 21 & -6427649341 & 6427649341 \\
\hline 2659 & 13 & 371447 & 371447 \\
\hline 2683 & 5 & 15 & $3 \cdot 5$ \\
\hline 2699 & 15 & 1378519456 & $2^{5} \cdot 43 \cdot 1001831$ \\
\hline 2707 & 7 & -4901 & $13^{2} \cdot 29$ \\
\hline 2731 & 11 & -1092 & $2^{2} \cdot 3 \cdot 7 \cdot 13$ \\
\hline 2803 & 9 & 29181 & $3 \cdot 71 \cdot 137$ \\
\hline 2819 & 21 & -5427534418429 & $23 \cdot 37 \cdot 6377831279$ \\
\hline 2843 & 15 & 4551355173 & $3 \cdot 1517118391$ \\
\hline 2851 & 11 & -25563 & $3 \cdot 8521$ \\
\hline 2939 & 29 & 1677457225439091 & $3^{4} \cdot 31 \cdot 151 \cdot 33937 \cdot 130363$ \\
\hline 2963 & 13 & -96843276 & $2^{2} \cdot 3^{6} \cdot 33211$ \\
\hline 2971 & 11 & 95873 & 95873 \\
\hline
\end{tabular}




\begin{tabular}{|c|c|c|c|c|c|}
\hline & \multirow{5}{*}{$p$} & & $p$ & \multirow{6}{*}{$\begin{array}{l}\mathfrak{f} \\
\mathfrak{p}_{2} \cdot \mathfrak{p}_{179} \\
\mathfrak{p}_{2}^{2} \cdot \mathfrak{p}_{11} \\
\mathfrak{p}_{2} \cdot \mathfrak{p}_{3}^{2} \cdot \mathfrak{p}_{5} \\
\mathfrak{p}_{2} \cdot \mathfrak{p}_{3} \cdot \mathfrak{p}_{41} \\
\mathfrak{p}_{2}^{3} \cdot \mathfrak{p}_{19}\end{array}$} \\
\hline & & & & 251 & \\
\hline & & & & 467 & \\
\hline & & & & 587 & \\
\hline & & & $\mathfrak{f}$ & 811 & \\
\hline & & \multirow{2}{*}{$\begin{array}{l}131 \\
179\end{array}$} & \multirow{2}{*}{$\begin{array}{l}\mathfrak{p}_{2} \cdot \mathfrak{p}_{3} \\
\mathfrak{p}_{2}^{2} \cdot \mathfrak{p}_{5}\end{array}$} & 827 & \\
\hline$p$ & $\mathfrak{f}$ & & & 859 & \\
\hline 59 & $\mathfrak{p}_{3}$ & 227 & & 1171 & $\mathfrak{p}_{23}$ \\
\hline 83 & $\mathfrak{p}_{5}^{\prime}$ & 523 & $\mathfrak{n}^{\prime}$ & 1483 & $\mathfrak{p}_{2}^{3}$ \\
\hline 139 & (1) & 571 & $\mathfrak{p}_{11}^{2}$ & 1523 & $\mathfrak{p}_{5} \cdot \mathfrak{p}_{7} \cdot \mathfrak{p}_{71}$ \\
\hline 211 & $\mathfrak{p}_{3}$ & 619 & $p_{2}$ & 1627 & $\mathfrak{p}_{3}^{2} \cdot \mathfrak{p}_{83}$ \\
\hline 283 & & 683 & $\mathfrak{p}_{31}$ & 1787 & $\mathfrak{p}_{2} \cdot \mathfrak{p}_{971}$ \\
\hline 307 & $\mathfrak{p}_{2}^{\prime 2} \cdot \mathfrak{p}_{2}^{\prime \prime}$ & 691 & $\mathfrak{p}^{\prime}$ & 1987 & $\mathfrak{p}_{3} \cdot \mathfrak{p}_{769}$ \\
\hline 379 & & 787 & $\mathfrak{p}_{3} \cdot \mathfrak{p}_{5}$ & 2011 & $\mathfrak{p}_{2} \cdot \mathfrak{q}_{3,3}^{\prime}$ \\
\hline 499 & & 947 & $\mathfrak{p}_{3} \cdot \mathfrak{p}_{5}$ & 2083 & $\mathfrak{p}_{11} \cdot \mathfrak{p}_{23} \cdot \mathfrak{p}_{53}$ \\
\hline 547 & $\mathfrak{p}_{2}^{\prime} \cdot \mathfrak{p}_{2}^{\prime \prime} \cdot \mathfrak{p}_{3}$ & 1747 & $\mathfrak{p}_{2} \cdot \mathfrak{p}_{5} \cdot \mathfrak{p}_{19}$ & $\begin{array}{l}2179 \\
2251\end{array}$ & $\mathfrak{p}_{2}^{2} \cdot \mathfrak{p}_{3}^{3}$ \\
\hline 883 & & 1867 & $\mathfrak{p}_{3} \cdot \mathfrak{p}_{7} \cdot \mathfrak{p}_{29}^{\prime}$ & $\begin{array}{l}2251 \\
2467\end{array}$ & $\mathfrak{p}_{2}^{4} \cdot \mathfrak{p}_{3}^{2} \cdot \mathfrak{p}_{7}$ \\
\hline 907 & $\mathfrak{p}_{5}^{\prime 2}$ & 2203 & $\mathfrak{p}_{2} \cdot \mathfrak{p}_{11} \cdot \mathfrak{p}_{13}$ & $\begin{array}{l}2401 \\
3019\end{array}$ & $\mathfrak{p}_{19} \cdot \mathfrak{p}_{43} \cdot \mathfrak{p}_{89}$ \\
\hline & $h=3$ & 2347 & $\mathfrak{p}_{5} \cdot \mathfrak{p}_{127}$ & $\begin{array}{l}3019 \\
3067\end{array}$ & $\begin{array}{l}\mathfrak{l}_{15373} \\
\mathfrak{p}^{4} \cdot \mathfrak{p}_{56 \mathcal{2}}\end{array}$ \\
\hline & & 2683 & $\mathfrak{p}_{3} \cdot \mathfrak{p}_{5}$ & 3187 & $\mathfrak{n}_{2}$ \\
\hline & & & $h=5$ & 3907 & $\mathfrak{n}^{8} \cdot \mathfrak{n}_{2} \cdot \mathfrak{n}^{2}$ \\
\hline & & & & 4603 & $\mathfrak{p}_{3}^{2} \cdot \mathfrak{p}_{83}^{\prime}$ \\
\hline & & & & 5107 & $\mathfrak{p}_{2} \cdot \mathfrak{p}_{7}^{2} \cdot \mathfrak{p}_{673}$ \\
\hline & & & & 5923 & $\mathfrak{p}_{11} \cdot \mathfrak{p}_{14879}$ \\
\hline & & & & & $h=7$ \\
\hline
\end{tabular}

\section{References}

[Ar] N. Arthaud, Thesis, Stanford University, 1987.

[B] C. Batut, D. Bernardi, H. Cohen, M. Olivier, GP/PARI Calculator, ftp://megrez.math.u-bordeaux.fr.

[BG] J. Buhler and B.H. Gross, Arithmetic on elliptic curves with complex multiplication II, Invent. Math. 79 (1985), 11-29.

[CW] J. Coates and A. Wiles, On the conjecture of Birch and Swinnerton-Dyer, Invent. Math. 39 (1977), 223-251.

[Fo] G. Folland, Harmonic analysis in phase space, Annals of Math Studies 122, Princeton University Press, Princeton, NJ, 1989.

[Gr1] B.H. Gross, Arithmetic on elliptic curves with complex multiplication, Lecture Notes in Mathematics 776, Springer-Verlag, Berlin-Heidelberg-New York, 1979.

[Gr2] B.H. Gross, On the conjecture of Birch and Swinnerton-Dyer for elliptic curves with complex multiplication in "Conference on Fermat's Last Theorem" pp. 219-236, Birkhauser, Boston, 1982.

[Gr3] B.H. Gross, Minimal models for elliptic curves with complex multiplication, Compositio Math. 45 (1982), 155-164.

[Ha] F. Hajir, On the class number of Hilbert class fields, Pacific J. Math. 181 (1997), 177-189. 
[H] F. Hajir, On units related to the arithmetic of elliptic curves with complex multiplication, Arch. Math. (Basel) 66 (1995), 280-291.

[HRV] F. Hajir and F. Rodriguez Villegas, Explicit elliptic units I, Duke Math. J. 90 (1997), $256-271$.

[He] E. Hecke, Zur Theorie der elliptischen Modulfunktionen, Math. Ann. 97 (1926), 210-242; also in Mathematische Werke, Vandenhoeck \& Ruprecht, Göttingen, 1970, $\S 23$.

[Iw] K. Iwasawa, A note on ideal class groups, Nagoya Math. J. 27 (1966), 239-47.

[Ma] Y. I. Manin, Cyclotomic fields and modular curves, Russian Math. Surveys 26 (1971), $7-78$.

[Mi] J. S. Milne, On the arithmetic of abelian varieties, Invent. Math. 17 (1972), 177-190.

[RV] F. Rodriguez Villegas, On the square root of special values of certain L-series, Invent. Math. 106 (1991), 549-573.

[RVY] F. Rodriguez Villegas and T. Yang, Central values of Hecke L-functions of CM number fields, preprint.

[RVZ] F. Rodriguez Villegas and D. Zagier, Square roots of central values of Hecke L-series, Advances in Number Theory (Kingston, ON, 1991), pp. 81-99, Oxford Sci. Publ., Oxford University Press, New York, 1993.

[Ru1] K. Rubin, Elliptic curves with complex multiplication and the conjecture of Birch and Swinnerton-Dyer, Invent. Math. 64 (1981), pp. 455-470.

[Ru2] K. Rubin, The "main conjectures" of Iwasawa theory for imaginary quadratic fields, Invent. Math. 103 (1991), 25-68.

[Sh] G. Shimura, On the zeta-function of an abelian variety with complex multiplication, Ann. of Math. 94 (1971), 504-533.

[dS] B. de Smit, A differential criterion for complete intersections, Journées Arithmétiques, Barcelona, 1995, Collect. Math. 48 (1997), 85-96.

Dept. of Mathematics, UCLA, Los Angeles, CA 90095

E-mail address: fhajir@math.ucla.edu

Dept. of Mathematics, California State University, San Marcos, San Marcos, CA 92096

E-mail address: fhajir@csusm.edu

Dept. of Mathematics, Princeton University, Princeton, NJ 08544

E-mail address: villegas@math.princeton.edu

Dept. of Mathematics, University of Texas, Austin, TX 78712

E-mail address: villegas@math.utexas.edu 